\title{
C. The Saturation of Colors.
}

BY L. M. Solomons.

The experiments of which a provisional account is given here were the outgrowth of an effort to determine whether least perceptible differences of color saturation obeyed Weber's law, and though they have branched out into the wider field of the general relation of white and black to the colors they are still best presented from this point of view.

In any color mixture we may distinguish two kinds of intensity: the intensity of coloring, and general light intensity. For example, if we take a red disk and compare it with a color wheel containing a large amount of white and a little red, the merest novice at color judgments will say that the red disk is the more intense red, while the wheel possesses greater general light intensity. To the former element, the intensity of coloration, we give the name saturation, reserving intensity for general light intensity.

Now, if in a color wheel we increase the amount of color, we change in general both the saturation and the intensity. Therefore in determining least perceptible differences (which will hereafter be denoted by the abbreviation L. P. D.) it is necessary to make sure that we are not judging by intensity. Our first plan was to mix the color-red-with a gray of the same intensity, so that increasing the red decreased the white, thus keeping the intensity constant. These experiments gave no very satisfactory results, though the failure to obey Weber's law was manifest. The reason soon became clear. When we increase the red we decrease the white. Now to assume that the saturation increment is measured by the increase of red is to assume that the saturation of a mixture depends only on the amount of color, and not at all upon the amount of white. This is not true.

If we take two color wheels putting in one, say $180^{\circ}$ red and 180 black, and in the other $180^{\circ}$ red and 180 white, the former appears very much more saturated than the latter, though the actual amount of red is the same. With such large differences 
as in the above example the difference of saturations is obvious to anyone. But to compare two mixtures of very different intensity with regard to their saturation, with any degree of accuracy, seems at first almost a hopeless task. But with a little practice, beginning with large differences and working down, the judgment becomes quite possible, and eventually exceedingly accurate. Owing to the training required the experiments were made only by Miss Stein and the writer.

The result of a long series of observations showed that the saturation of a mixture of color and white is entirely independent of the intensity, and of the actual quantity of color, and depends only on the ratio of the color to the white. The law is perfectly obeyed within the limits of experimental error (a few degrees). The equality point was always determined by the method of least observable difference, though it was not long before the judgment of the equality point became more accurate than in most judgments, being nearly always placed in the same position, for movements in both directions. The colors used were red and blue. The teleological significance of the law is obvious. It enables us to identify objects in varying light intensity. The characteristic of a colored object is the proportion of the colored light to the white light that it reflects. The actual quantity of colored light depends upon the intensity of the incident light. It is therefore of the greatest importance for the recognition of objects that the intensity of coloration should depend upon the ratio of colored light to white, and not upon the actual quantity of colored light.

Meantime a series of measurements of L. P. D. made out the following facts: For a constant saturation the L. P. D. is constant measured in terms of actual amount of color added, that is, if in a mixture of $50^{\circ}$ white and $50^{\circ}$ red the red must be increased by $4^{\circ}$ to give a L. P. D; in a mixture of $100^{\circ}$ red and $100^{\circ}$ white, the red must also be increased by $4^{\circ}$; secondly, the L. P. D. increases with the saturation. To find out the exact law of increase it is necessary to have a measure of saturation.

By direct observation we only determine when two saturations are equal. Now the law that they are equal when the 
ratios of the color to the white are equal admits of more than one interpretation. For when the ratios of color to white are equal the ratios of color to white + color or any proportion thereof, as white $+1 / 2$ color, are also equal. Calling $S$ the saturation, we have the general formula $S=\frac{c}{w+a c}$ satisfying the law of equality of saturation for all values of a. We have seen that for constant saturation the saturation increment for a L. P. $D$. varied inversely as the intensity-for the actual color increment being constant, the saturation increment corresponding to it will vary inversely as the total quantity of light. Assuming it to vary directly as the saturation, we should have the formula $\Delta S\left[=\frac{\Delta c}{W+a c}\right]=\frac{c}{W+a c} I$, I being the intensity, that is, the actual increment of color, $\Delta c$, varies directly as the ratio of color to intensity. Since the result is independent of the quantity $\mathrm{W}+$ ac it might seem preferable to give the law the simple, verifiable formulation $d c=\frac{c}{\mathrm{I}}$, and from a physical standpoint this would of course be preferable. But psychologically it is bad because the quantity $c$ has no psychological equivalent. The psychical fact, intensity of coloration, depends upon a physical ratio $-\frac{c}{w+a c}$. If we are to keep to psychical facts we must use the quantities saturation and intensity. Remembering therefore that $\Delta \mathrm{c}=\frac{\mathrm{c}}{\mathrm{I}}$ is the best expression of the observed physical fact it is yet well, I think, to retain the somewhat hypothetical formula $\Delta S=\frac{S}{I}$, as more suggestive from the psychological point of view.

As to the accuracy with which the law $\Delta \mathrm{c} \propto \frac{\mathrm{c}}{\mathrm{I}}$ is obeyed, many difficulties have arisen in the effort to fully verify it. Several very short series of observations have obeyed it within the limits of experimental error. In attempting to get long series of observations it was found that owing to the constant increase of skill in the subject, as well as other causes of variation, the different parts of the series are not strictly comparable. By planning the series with these facts in view, however, accurate results may I think be obtained.

The above L. P. D. law contains two anomalies which require investigation. The first is that though the saturation in- 
crement varies inversely as the intensity when the change is produced by increasing the proportion of color and white in the wheel, it is not affected, at least not to any easily observable extent, by variations in the intensity of the incident light. The other is that if we adopt the usual conception of a L. P. D., viz., that it represents a simple increment of sensation, the L. P. D. law contradicts the saturation law. For if we call the sensation of saturation $S$ and the physical quantity corresponding to it $\left(\frac{\mathrm{c}}{\mathrm{w}+\mathrm{ac}}\right) \mathrm{s}$, we have the law $\mathrm{dS}=\frac{\mathrm{ds}}{\mathrm{s}} \mathrm{I}$

By integration this formula gives $S=I$ logs, which contradicts the saturation law that $S$ depends on s only and is independent of I. A similar contradiction exists in the other formulation of the law.

The explanation of the above brings up two questions. What is the general relation between intensity and color quality, and what is the real significance of a L. P. D.?

A number of experiments were carried out in connection with the former problem, most of which have no immediate bearing on the subject in hand. I wish to describe only one series, the results of which are important. An apparatus was arranged, whereby two color wheels were placed in lights of different intensities. The arrangement was a very simple one, the wheels being placed opposite a window divided into two portions by a vertical board. By placing a screen between the two wheels perpendicular to their plane and that of the window, each wheel received light only from its own side of the window. The subject sat in front of the board dividing the window and had both wheels well in view. By varying the size of the openings the light could be varied at pleasure.

Place a white disk in a weak light, and a black and white in a strong light. It is not possible, by varying the proportion of black and white in the well-lit disk to get the two to look alike. It is possible to get them of the same general light intensity, or of the same shade of gray, but not both together. When the light intensity is the same the well-lit disk is a very dark gray and the other a white, dimly seen. When of the same shade, the well-lit disk is very much more intense. It is the same with colors. A blue disk is seen distinctly as a pure blue, even 
when the light is so feeble as to make it scarcely visible, while a blue and black disk appears a dark navy blue, no matter how strong the light. There is much individual difference here. A white disk in weak light appeared much more like a gray to Miss Stein than to me, but in no way could either of us get equality between the strong and weak light wheels. It should perhaps be stated that these experiments were first carried out with the object of really securing such an equality, and our inability to do so was a serious inconvenience; so that the result was anything but desired by us. We made every effort to see the disks alike.

If, however, we look at the disks through black tubes, held to the eye so as to shut out everything else from the field of view, there is no trouble about perfect equality. The white disk in dim light looks gray, the blue, navy blue, etc.

The conclusions are obvious. Intensity as such does not affect color quality at all. It remains a separate and distinct element in every color presentation. Blackness cannot be regarded as the inverse of intensity, nor as a sensational element at all. For it depends not upon the character of the light coming from the given body, but upon its relation to the immediate field of view. It must be regarded as an element added to every presentation by some reflex process, and giving the relation of the object to its immediate field of view-or to the incident light. It is not a mere question of comparison with other objects, for in all the above experiments there were two objects seen, yet the most intense disk was also the blackest. Nor was it simply a question of seeing objects 'as we know them to be,' instead of as they appear. For in our efforts to obtain equality all sorts of variations were made in the proportion of black and white and color in the two disks, of which the subject was unaware; yet it was not possible to get equality as long as the two disks were seen in different backgrounds. The teleological significance of the law is obvious. It makes blackness a 'body property,' independent of the intensity of the illumination.

This compels us to adopt a four-fold, instead of the usual three-fold, representations of colored objects. They can vary in four independent ways : I. color quality, or tone ; 2. saturation; 
3. intensity; 4. blackness. Any one of these may be made to vary while the others remain constant. This is a purely psychological classification of course, giving the different subjective effects which a colored object produces. That color quality may vary, the other elements remaining constant, is clear theoretically, though to actually compare the saturation of different colors is difficult. Saturation may be made to vary independently by simply changing the proportion of color to white, while keeping the sum of their intensities constant. Intensity by simply increasing the incident light, and blackness by increasing the incident light and at the same time decreasing the amount of color and white in the disk so as to keep the intensity constant, while its relation to the intensity of the field changes. When the saturation of any color becomes zero we call it a gray, and grays may vary in intensity and blackness. The above four elements, and no fewer, completely describe any color combination. White is not given explicitly, but saturation and intensity together determine the amount of white, if whiteness is different from intensity, so that the above formulation is entirely independent of all special color theories. The general result of all this, it will be noticed, is to accentuate the subjective aspect of color theory.

We can now understand the law of L. P. D. of saturation. Consider for a moment the process of making a judgment of saturation. Suppose we have one disk of $40^{\circ}$ red and $20^{\circ}$ white, and another $120^{\circ}$ red and $60^{\circ}$ white. The only difference between them is in blackness and intensity. The intensity, however, is easily abstracted from. It does not 'fuse' into the general presentation but remains as a fairly distinct element. The black, however, is an organic part of the percept bound up with the rest. The process of perceiving the two disks to be equal is abstracting from the black element. Once able to separate that, and they are seen equal. The training required for judging saturation is simply the training in isolating the black element in a color presentation. Our experience amply confirms this theoretical deduction. This has actually been the difficulty encountered in making judgments, and our records are full of such notes as judgment uncertain 
on account of inability to separate black'-notes taken, it should be stated long before their theoretical significance was suspected.

It is clear now why the L.P. D. varies inversely as the quantity of color and white in the disk, but not as the intensity of the incident light. Changing the intensity in the first way changes the blackness, while changing it in the second way does not. The law should really be stated $\triangle \mathrm{S} \propto \mathrm{SB}$ where $\mathrm{B}$ is blackness. If we regard the $\mathrm{L}$. P. D. as measuring primarily the ease or difficulty of a judgment, then we can understand why it varies directly as the amount of black. The process of isolating the black becomes the more difficult as the amount of black becomes greater-as the black becomes a more prominent feature of the presentation. The ordinary conception of a L. P. D. leads to a contradiction in the case of saturation because we have not here the simple case of comparing two quantities; but there is another process to be gone through in addition to the primary judgment-the isolation of the black. It is necessary therefore to go back to the primary significance of a L. P. D. in order to properly understand the law.

We have begun a series of experiments on the effect of tiring on saturation. The results are very encouraging, but as yet too few to permit of much theorizing. On tiring with white, the saturation of a color is increased by a constant proportion of its value for the same time of tiring. The increase seems to be proportional to the time of tiring for the times tried-5 to I5 seconds-but the experiments have not gone far enough yet to give more than provisional results.

\section{Fluctuations of the Attention (I.).}

By J. B. HyLAN.

The facts of the oscillation of feeble impressions are still under discussion. The alternate increase and decrease of weak sensations may be of peripheral or of central origin; the peripheral sources may be nervous or muscular, the central process may go on in the cortical end apparatus of the sensory nerves or 